\title{
Convex data modelling using rational bi-cubic spline function
}

\author{
Muhammad Abbas ${ }^{\mathrm{a}, \mathrm{b}, *}$, Ahmad Abd Majid ${ }^{\mathrm{b}}$, Mohd Nain Hj Awang ${ }^{\mathrm{c}}$, Jamaludin Md Ali ${ }^{\mathrm{b}}$ \\ a Department of Mathematics, University of Sargodha, 40100 Sargodha, Pakistan \\ b School of Mathematical Sciences, Universiti Sains Malaysia, 11800 USM Penang, Malaysia \\ c School of Distance Education, Universiti Sains Malaysia, 11800 USM Penang, Malaysia
}

*Corresponding author, e-mail: m.abbas@uos.edu.pk

\begin{abstract}
A piecewise rational cubic function in the form of a cubic/quadratic has been extended to a rational bicubic blended function to visualize 3D convex data. It involves twelve shape parameters in each rectangular mesh for its representation. Simple data-dependent constraints are imposed on four shape parameters to conserve convexity of convex data. The remaining shape parameters are left up to the user for modification of convex surfaces. Several numerical examples are presented to show the visually pleasing convexity preserving surfaces as compared to existing interpolants.
\end{abstract}

KEYWORDS: convex surfaces, rational bi-cubic blended function, 3D convex data, shape parameters

\section{INTRODUCTION}

Data visualization is the study of visual display of data. The goal of visualization is to leverage existing scientific methods by providing new scientific insight through visual methods. Large amount of data is transformed into graphs and figures either to complete underlying information from geography, metrology, mining, basic sciences, medicine or to communicate human imagination like aerospace industries, architecture, fine arts, advertising, education by visualization tools. For visualization of data, there are three shape features of data named as positivity, monotonicity and convexity. These shape features are necessary to be conserved to understand the meaning of underlying physical phenomenon and to transform the idea of designer to reality. The objective of the present study is to visualize the $3 \mathrm{D}$ convex data and conserve its inherited shape features. The significance of the convexity preserving interpolation problems in industry cannot be denied. A crumpled surface is an unwanted characteristic. Human aesthetic sense demands convexity preserving nice and smooth surfaces without wiggles. A number of examples can be listed in this area.

(i) Designing well shaped smooth surfaces arise in manufacturing the TV-screens. In order to accomplish with the demands of the customer, as flat as possible TV-screens are most appreciated. In the surface designing sense we can say that the screens must preserve the convexity ${ }^{1}$. (ii) Convexity plays significant role in the modelling of cars in automobile industry, aero-plane and ship design because human aesthetic sense always demands curves and surfaces without undulations. A great deal of research on data visualization and shape preserving has been done. Some rational cubic $^{2}$ and rational quadratic functions have been extended to rational bi-cubic blended ${ }^{3}$ and rational bi-quadratic blended ${ }^{4}$ interpolants to visualize the $3 \mathrm{D}$ convex data in rectangular mesh case. The rational bi-cubic blended function is simpler, easy to compute and implement and computational economical because it conserves the shape of 3D convex data with fewer constraints imposed on shape parameters as compared to rational bi-cubic or bi-quartic functions. These rational schemes have common characteristics in a way that local and no extra knots are inserted in the interval where interpolants lost the inherited shape properties. In contrast, firstly, a piecewise cubic Bernstein-Bézier polynomial function ${ }^{5}$, quadratic spline interpolation ${ }^{6}$, a piecewise quadratic polynomial $^{7}$, and cubic Hermite interpolation ${ }^{8}$ have been used to solve the problem of interpolating monotone and convex data in the sense of monotonicity and convexity preserving schemes which were very economical but the methods generally inserted extra knots in the interval to visualize and conserve the shape of data. Secondly, Costantini ${ }^{9}$ developed a global scheme for preservation of convex surfaces on rectangular grid. The scheme ${ }^{10}$ has some research gaps like the degree of interpolant in some rectangular 
patches which was too large, and the resulting surfaces were not visually pleasing and smooth. Casciola and Romani ${ }^{11}$ have extended NURBS to rational interpolating spline with tension parameters for rectangular grid case. Carnicer, Garcia-Esnaola and Peña ${ }^{12}$ analysed the convexity preserving properties of rational Bézier and NURBS curves from a geometric point of view, and also characterized totally positive systems of functions in terms of geometric convexity preserving properties of the rational curves.

Dodd, McAllister and Roulier ${ }^{13}$ have developed quadratic splines to conserve the convexity of surface in rectangular case. The scheme guaranteed the shape along the boundary of each rectangular grid but it does not conserve the shape in the interior of the grids. The scheme produced undesirable flat spots due to vanishing of second order mixed partial derivatives on the boundary of the rectangles.

In this work, a $C^{1}$ piecewise rational cubic function with three shape parameters ${ }^{2}$ is extended to rational bi-cubic blended function. It addresses the problem of constructing convexity preserving surfaces through 3D convex data in rectangular case. It improves on existing interpolants in following way:

(i) In Ref. 4, the degree of smoothness of convex surface interpolant is $C^{0}$ while in this study the degree of smoothness is $C^{1}$.

(ii) The present scheme is uniformly valid for the data with derivatives or without derivatives. In contrast, the schemes developed in Ref. 14 did not maintain the shape of data when data are given with derivatives at knots.

(iii) There is no need of extra knots in the interval for conservation of convex surfaces. In contrast, the schemes ${ }^{5,6}$ achieve the required shape of data by inserting extra knots in the interval where the functions lose convexity.

(iv) The present scheme is tested through several numerical examples and it is found to be local in comparison with global schemes ${ }^{9}$, time saving and computational economical due to second degree of denominator in rational function in comparison the function ${ }^{3}$ has cubic denominator and produces smooth and visually pleasing surfaces than existing schemes ${ }^{4}$.

\section{REVIEW OF RATIONAL CUBIC FUNCTION}

In this section, a piecewise rational cubic function ${ }^{2}$ in (cubic/quadratic) form is proposed which is different from rational cubic functions ${ }^{3}$. We choose this function due to low computational cost because the second order derivative of the functions ${ }^{3}$ for the calculation of convexity, become a rational function of degree 7 while the second order derivative of proposed function provides a rational function of degree 5 .

Let $\left\{\left(t_{i}, f_{i}\right), i=0,1,2, \ldots, n\right\}$ be the given set of data points such as $t_{0}<t_{1}<t_{2}<\ldots<$ $t_{n}$. The piecewise rational cubic function with three shape parameters ${ }^{2}$, in each subinterval $\left[t_{i}, t_{i+1}\right]$, $i=0,1,2, \ldots, n-1$ can be defined as

$$
R_{i}(t)=\frac{P_{i}(t)}{Q_{i}(t)}
$$

where

$$
\begin{aligned}
P_{i}(t) & =a_{i} f_{i}(1-\omega)^{3} \\
& +\left[f_{i}\left(2 a_{i}+b_{i}+c_{i}\right)+a_{i} h_{i} d_{i}\right] \omega(1-\omega)^{2} \\
& +\left[f_{i+1}\left(a_{i}+b_{i}+2 c_{i}\right)-c_{i} h_{i} d_{i+1}\right] \omega^{2}(1-\omega) \\
& +c_{i} f_{i+1} \omega^{3} \\
Q_{i}(t) & =a_{i}(1-\omega)^{2}+\left(a_{i}+b_{i}+c_{i}\right) \omega(1-\omega)+c_{i} \omega^{2},
\end{aligned}
$$

when $\omega=\frac{\left(t-t_{i}\right)}{h_{i}}, h_{i}=t_{i+1}-t_{i}$ and $a_{i}, b_{i}$ and $c_{i}$ are positive shape parameters. A piecewise rational cubic function (1) has attained $C^{1}$ continuity by using the following conditions:

$$
\begin{array}{ll}
R_{i}\left(t_{i}\right)=f_{i}, & R_{i}\left(t_{i+1}\right)=f_{i+1}, \\
R_{i}^{\prime}\left(t_{i}\right)=d_{i}, & R_{i}^{\prime}\left(t_{i+1}\right)=d_{i+1},
\end{array}
$$

where $d_{i}$ denote the derivative values that are used for smoothness of required curve and calculated by arithmetic mean method and $R_{i}^{\prime}(t)$ denotes the first order derivative with respect to $t$. A piecewise rational cubic function (1) becomes a standard cubic Hermite spline when we set the values of shape parameters as $a_{i}=1, b_{i}=0$ and $c_{i}=1$. The following result developed in Ref. 2 as

Theorem 1 (Ref. 2) A piecewise rational cubic function (1) conserves the convexity of convex data, if in each subinterval $\left[t_{i}, t_{i+1}\right], i=0,1,2, \ldots, n-1$, the shape parameters satisfy the following conditions

$$
\begin{aligned}
& a_{i}>0, \quad c_{i}>0 \\
& b_{i}>\max \left\{0, \frac{c_{i}\left(d_{i+1}-\Delta_{i}\right)}{\left(\Delta_{i}-d_{i}\right)}, \frac{a_{i}\left(\Delta_{i}-d_{i}\right)}{\left(d_{i+1}-\Delta_{i}\right)}\right\},
\end{aligned}
$$

where $\Delta_{i}=\frac{f_{i+1}-f_{i}}{h_{i}}$. The above result can be rearranged as

$$
\begin{aligned}
& a_{i}>0, \quad c_{i}>0 \\
& b_{i}=r_{i}+\max \left\{0, \frac{c_{i}\left(d_{i+1}-\Delta_{i}\right)}{\left(\Delta_{i}-d_{i}\right)}, \frac{a_{i}\left(\Delta_{i}-d_{i}\right)}{\left(d_{i+1}-\Delta_{i}\right)}\right\}
\end{aligned}
$$

for some $r_{i}>0$. 
Table 12 D convex data set.

\begin{tabular}{ccccccc}
\hline$i$ & 1 & 2 & 3 & 4 & 5 & 6 \\
\hline$t_{i}$ & -8 & -7 & 2.2 & 7 & 10 & 12 \\
$f_{i}$ & 4.5 & 4 & 3.55 & 4 & 4.5 & 5 \\
\hline
\end{tabular}

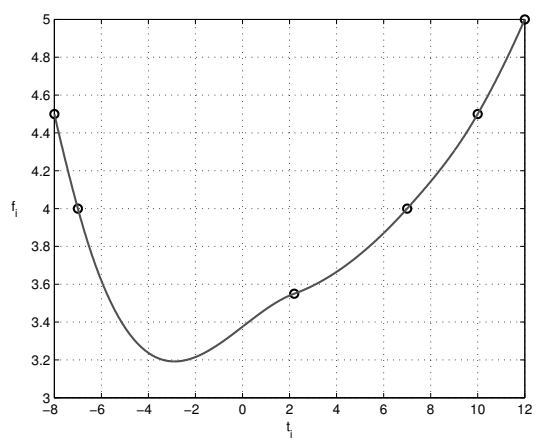

Fig. 1 Cubic Hermite spline curve.

Example 1 A convex data set is taken in Table 1. Fig. 1 is generated by cubic Hermite spline scheme Ref. 15 that does not conserve the convexity. Fig. 2 is generated by using Piecewise Cubic Hermite Interpolating Polynomial (PCHIP, Built-in MATLAB program) to conserve the convexity of same data but the visual model is not smooth at some data points. On the other hand, Fig. 3 and Fig. 4 are generated by rational cubic function (1) using Theorem 1 to conserve the convexity. The effect of shape parameters can be seen by noting the difference in $C^{1}$ smoothness of these convexity preserving curves in Fig. 2, Fig. 3, and Fig. 4.

\section{RATIONAL BI-CUBIC BLENDED SPLINE FUNCTION}

This section introduces a $C^{1}$ rational bi-cubic blended function with shape parameters for $3 \mathrm{D}$ convex data modelling and preserves the convexity of surfaces

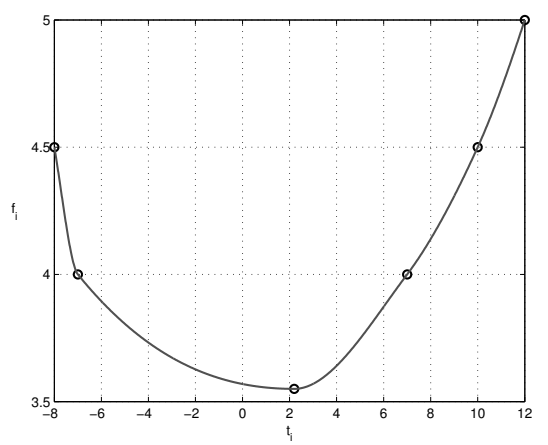

Fig. 2 PCHIP curve.

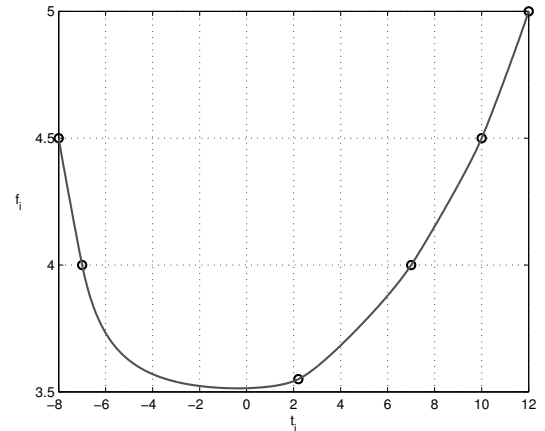

Fig. 3 Convexity-preserving $C^{1}$ curve using rational cubic function with $a_{i}=c_{i}=0.6$ and $r_{i}=0.01$.

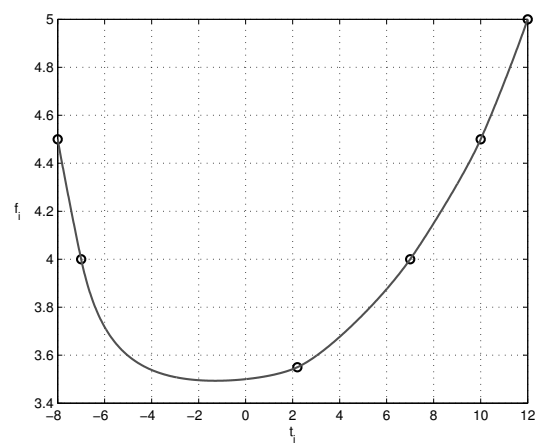

Fig. 4 Convexity-preserving $C^{1}$ curve using rational cubic function with $a_{i}=c_{i}=1.0$ and $r_{i}=0.1$.

of 3D convex data. The rational bi-cubic blended function is suitable to arrange the data over rectangular mesh $\left[\xi, \xi_{1}\right] \times\left[\sigma, \sigma_{1}\right]$ such that $\xi=t_{0}<t_{1}<$ $\ldots<t_{n}=\xi_{1}$ and $\sigma=u_{0}<u_{1}<\ldots<u_{m}=\sigma_{1}$. The rational bi-cubic blended function over each patch $\left[t_{i}, t_{i+1}\right] \times\left[u_{j}, u_{j+1}\right], i=0,1,2, \ldots, n-1$, $j=0,1,2, \ldots, m-1$ is defined as

$$
\begin{aligned}
& R(t, u)= {\left[m_{0}(\omega) m_{1}(\omega)\right]\left[\begin{array}{c}
R\left(t_{i}, u\right) \\
R\left(t_{i+1}, u\right)
\end{array}\right] } \\
&+\left[n_{0}(\psi) n_{1}(\psi)\right]\left[\begin{array}{c}
R\left(t, u_{j}\right) \\
R\left(t, u_{j+1}\right)
\end{array}\right] \\
&-\left[m_{0}(\omega) m_{1}(\omega)\right]\left[\begin{array}{cc}
F_{i, j} & F_{i, j+1} \\
F_{i+1, j} & F_{i+1, j+1}
\end{array}\right]\left[\begin{array}{l}
n_{0}(\psi) \\
n_{1}(\psi)
\end{array}\right]
\end{aligned}
$$

with

$m_{0}(\omega)=(1-\omega)^{2}(1+2 \omega), \quad m_{1}(\omega)=\omega^{2}(3-2 \omega)$, $n_{0}(\phi)=(1-\psi)^{2}(1+2 \psi), \quad n_{1}(\psi)=\psi^{2}(3-2 \psi)$

are called Hermite blending functions with $\omega=\frac{t-t_{i}}{h_{i}}$, $\psi=\frac{u-u_{j}}{\hat{h}_{j}}$ where $h_{i}=t_{i+1}-t_{i}, \hat{h}_{j}=u_{j+1}-u_{j}$. 
The $R\left(t, u_{j}\right), R\left(t, u_{j+1}\right), R\left(t_{i}, u\right)$ and $R\left(t_{i+1}, u\right)$ are four boundary rational cubic curves of rectangular patch which can be defined as

$$
R\left(t, u_{j}\right)=\frac{\sum_{i=0}^{3}(1-\omega)^{3-i} \omega^{i} \beta_{i}}{s_{1}(\omega)}
$$

with

$$
\begin{aligned}
\beta_{0}= & a_{i, j} F_{i, j} \\
\beta_{1}= & \left(2 a_{i, j}+b_{i, j}+c_{i, j}\right) F_{i, j}+a_{i, j} h_{i} F_{i, j}^{t} \\
\beta_{2}= & \left(a_{i, j}+b_{i, j}+2 c_{i, j}\right) F_{i+1, j}-c_{i, j} h_{i} F_{i+1, j}^{t}, \\
\beta_{3}= & c_{i, j} F_{i+1, j} \\
s_{1}(\omega)= & a_{i, j}(1-\omega)^{2}+c_{i, j} \omega^{2} \\
& +\left(a_{i, j}+b_{i, j}+c_{i, j}\right) \omega(1-\omega) \\
& R\left(t, u_{j+1}\right)=\frac{\sum_{i=0}^{3}(1-\omega)^{3-i} \omega^{i} \gamma_{i}}{s_{2}(\omega)}
\end{aligned}
$$

with

$$
\begin{aligned}
\gamma_{0}= & a_{i, j+1} F_{i, j+1}, \\
\gamma_{1}= & \left(2 a_{i, j+1}+b_{i, j+1}+c_{i, j+1}\right) F_{i, j+1} \\
& +a_{i, j+1} h_{i} F_{i, j+1}^{t}, \\
\gamma_{2}= & \left(a_{i, j+1}+b_{i, j+1}+2 c_{i, j+1}\right) F_{i+1, j+1} \\
& -c_{i, j+1} h_{i} F_{i+1, j+1}^{t}, \\
\gamma_{3}= & c_{i, j+1} F_{i+1, j+1}, \\
s_{2}(\omega)= & a_{i, j+1}(1-\omega)^{2}+c_{i, j+1} \omega^{2} \\
& +\left(a_{i, j+1}+b_{i, j+1}+c_{i, j+1}\right) \omega(1-\omega) ; \\
R & \left.t_{i}, u\right)=\frac{\sum_{i=0}^{3}(1-\psi)^{3-i} \psi^{i} \delta_{i}}{s_{3}(\psi)}
\end{aligned}
$$

with

$$
\begin{aligned}
\delta_{0}= & \hat{a}_{i, j} F_{i, j}, \\
\delta_{1}= & \left(2 \hat{a}_{i, j}+\hat{b}_{i, j}+\hat{c}_{i, j}\right) F_{i, j}+\hat{a}_{i, j} \hat{h}_{j} F_{i, j}^{u}, \\
\delta_{2}= & \left(\hat{a}_{i, j}+\hat{b}_{i, j}+2 \hat{c}_{i, j}\right) F_{i, j+1}-\hat{c}_{i, j} \hat{h}_{j} F_{i, j+1}^{u}, \\
\delta_{3}= & \hat{c}_{i, j} F_{i, j+1}, \\
s_{3}(\psi)= & \hat{a}_{i, j}(1-\psi)^{2}++\hat{c}_{i, j} \psi^{2} \\
& +\left(\hat{a}_{i, j}+\hat{b}_{i, j}+\hat{c}_{i, j}\right) \psi(1-\psi) ; \\
& R\left(t_{i+1}, u\right)=\frac{\sum_{i=0}^{3}(1-\psi)^{3-i} \psi^{i} \chi_{i}}{s_{4}(\psi)}
\end{aligned}
$$

with

$$
\begin{aligned}
\chi_{0}= & \hat{a}_{i, j+1} F_{i+1, j}, \\
\chi_{1}= & \left(2 \hat{a}_{i, j+1}+\hat{b}_{i, j+1}\right. \\
& \left.+\hat{c}_{i, j+1}\right) F_{i+1, j}+\hat{a}_{i, j+1} \hat{h}_{j} F_{i+1, j}^{u}, \\
\chi_{2}= & \left(\hat{a}_{i, j+1}+\hat{b}_{i, j+1}+2 \hat{c}_{i, j+1}\right) F_{i+1, j+1} \\
& -\hat{c}_{i, j+1} \hat{h}_{j} F_{i+1, j+1}^{u},
\end{aligned}
$$

$$
\begin{aligned}
\chi_{3}= & \hat{c}_{i+1, j} F_{i+1, j+1}, \\
s_{4}(\psi)= & \hat{a}_{i, j+1}(1-\psi)^{2}+\hat{c}_{i, j+1} \psi^{2} \\
& +\left(\hat{a}_{i, j+1}+\hat{b}_{i, j+1}+\hat{c}_{i, j+1}\right) \psi(1-\psi) .
\end{aligned}
$$

Here $F_{i, j}^{t}$ and $F_{i, j}^{u}$ are the approximations of the partial derivatives at given data points $F(i, j)$, $i=0,1,2, \ldots, n, j=0,1,2, \ldots, m$

\section{D CONVEX SURFACE INTERPOLATION}

In this section, we discuss the 3D convex modelling in the view of convexity preserving surface using rational bi-cubic blended function (3) with twelve shape parameters. Let $\left\{\left(t_{i}, u_{j}, F_{i, j}\right), i=0,1,2, \ldots, n ; j=\right.$ $0,1,2, \ldots, m\}$ be given 3D convex data. It is arranged on rectangular grid $\left[t_{i}, t_{i+1}\right] \times\left[u_{j}, u_{j+1}\right], i=$ $0,1,2, \ldots, n-1, j=0,1,2, \ldots, m-1$ such that

$$
\begin{aligned}
& F_{i, j}^{t}<F_{i+1, j}^{t}, \quad F_{i, j}^{u}<F_{i, j+1}^{u}, \\
& \Delta_{i, j}<\Delta_{i+1, j}, \quad \hat{\Delta}_{i, j}<\hat{\Delta}_{i, j+1}, \\
& \Delta_{i, j}<F_{i, j}^{t}<\Delta_{i+1, j}, \quad \hat{\Delta}_{i, j}<F_{i, j}^{u}<\hat{\Delta}_{i, j+1},
\end{aligned}
$$

where

$$
\Delta_{i, j}=\frac{F_{i+1, j}-F_{i, j}}{h_{i}}, \quad \hat{\Delta}_{i, j}=\frac{F_{i, j+1}-F_{i, j}}{\hat{h}_{j}} .
$$

The free parameters are

$$
\begin{aligned}
& a_{i, j}, a_{i, j+1}, \hat{a}_{i, j}, \hat{a}_{i+1, j}>0 \\
& c_{i, j}, c_{i, j+1}, \hat{c}_{i, j}, \hat{c}_{i+1, j}>0 .
\end{aligned}
$$

According to the result developed in Ref. 11, the rational bi-cubic blended surface patch inherits all the properties of network of boundary curves. Hence the rational bi-cubic blended function (3) to visualize the 3D convex data and conserves the convexity of convex data if the four boundary curves $R\left(t, u_{j}\right), R\left(t, u_{j+1}\right)$, $R\left(t_{i}, u\right)$, and $R\left(t_{i+1}, u\right)$ are defined in equations (4)(7) are convex. The boundary curve $R\left(t, u_{j}\right)$ is convex if $R^{\prime \prime}\left(t, u_{j}\right)>0$, i.e.,

$$
R^{\prime \prime}\left(t, u_{j}\right)=\frac{\sum_{k=0}^{5}(1-\omega)^{5-k} \omega^{k} \xi_{k}}{h_{i}\left(s_{1}(\omega)\right)^{3}}>0
$$

with

$$
\begin{aligned}
\xi_{0}= & 2 a_{i, j}^{2}\left[\left(a_{i, j}+c_{i, j}\right)\left(\Delta_{i, j}-F_{i, j}^{t}\right)\right. \\
& \left.+b_{i, j}\left(\Delta_{i, j}-F_{i, j}^{t}\right)-c_{i, j}\left(F_{i+1, j}^{t}-\Delta_{i, j}\right)\right], \\
\xi_{1}= & \xi_{0}+2 a_{i, j}^{2}\left[5 c_{i, j}\left(\Delta_{i, j}-F_{i, j}^{t}\right)+c_{i, j} F_{i, j}^{t}\right], \\
\xi_{2}= & \xi_{0}+6 a_{i, j}\left[c_{i, j}^{2}\left(F_{i+1, j}^{t}-\Delta_{i, j}\right)\right. \\
& \left.+2 a_{i, j} c_{i, j}\left(\Delta_{i, j}-F_{i, j}^{t}\right)\right],
\end{aligned}
$$




$$
\begin{aligned}
\xi_{3}= & \xi_{5}+6 c_{i, j}\left[a_{i, j}^{2}\left(\Delta_{i, j}-F_{i, j}^{t}\right)\right. \\
& \left.+2 a_{i, j} c_{i, j}\left(F_{i+1, j}^{t}-\Delta_{i, j}\right)\right], \\
\xi_{4}= & \xi_{5}+2 c_{i, j}^{2}\left[5 a_{i, j}\left(F_{i+1, j}^{t}-\Delta_{i, j}\right)+a_{i, j} F_{i+1, j}^{t}\right], \\
\xi_{5}= & 2 c_{i, j}^{2}\left[\left(a_{i, j}+c_{i, j}\right)\left(F_{i+1, j}^{t}-\Delta_{i, j}\right)\right. \\
& \left.+b_{i, j}\left(F_{i+1, j}^{t}-\Delta_{i, j}\right)-a_{i, j}\left(\Delta_{i, j}-F_{i, j}^{t}\right)\right],
\end{aligned}
$$

and $\sum_{k=0}^{5}(1-\omega)^{5-k} \omega^{k} \xi_{k}>0$ if $\xi_{k}>0$ for $k=0, \ldots, 5$. Thus $\xi_{k}>0$ if

$b_{i, j}>\max \left\{0, \frac{c_{i, j}\left(F_{i+1, j}^{t}-\Delta_{i, j}\right)}{\left(\Delta_{i, j}-F_{i, j}^{t}\right)}, \frac{a_{i, j}\left(\Delta_{i, j}-F_{i, j}^{t}\right)}{\left(F_{i+1, j}^{t}-\Delta_{i, j}\right)}\right\}$

or equivalently, for some $r_{i, j}>0$,

$$
\begin{array}{r}
b_{i, j}=r_{i, j}+\max \left\{0, \frac{c_{i, j}\left(F_{i+1, j}^{t}-\Delta_{i, j}\right)}{\left(\Delta_{i, j}-F_{i, j}^{t}\right)},\right. \\
\left.\frac{a_{i, j}\left(\Delta_{i, j}-F_{i, j}^{t}\right)}{\left(F_{i+1, j}^{t}-\Delta_{i, j}\right)}\right\} .
\end{array}
$$

Similarly, the boundary curve $R\left(t, u_{j+1}\right)$ is convex, if $R^{\prime \prime}\left(t, u_{j+1}\right)>0$, i.e.,

$$
R^{\prime \prime}\left(t, u_{j+1}\right)=\frac{\sum_{k=0}^{5}(1-\omega)^{5-k} \omega^{k} \zeta_{k}}{h_{i}\left(s_{2}(\omega)\right)^{3}}>0
$$

with

$$
\begin{aligned}
\zeta_{0}= & 2 a_{i, j+1}^{2}\left[\left(a_{i, j+1}+c_{i, j+1}\right)\left(\Delta_{i, j+1}-F_{i, j+1}^{t}\right)\right. \\
& +b_{i, j+1}\left(\Delta_{i, j+1}-F_{i, j+1}^{t}\right) \\
& \left.-c_{i, j+1}\left(F_{i+1, j+1}^{t}-\Delta_{i, j+1}\right)\right] \\
\zeta_{1}= & \zeta_{0}+2 a_{i, j+1}^{2}\left[5 c_{i, j+1}\left(\Delta_{i, j+1}-F_{i, j+1}^{t}\right)\right. \\
& \left.+c_{i, j+1} F_{i, j+1}^{t}\right] \\
\zeta_{2}= & \zeta_{0}+6 a_{i, j+1}\left[c_{i, j+1}^{2}\left(F_{i+1, j+1}^{t}-\Delta_{i, j+1}\right)\right. \\
& \left.+2 a_{i, j+1} c_{i, j+1}\left(\Delta_{i, j+1}-F_{i, j+1}^{t}\right)\right] \\
\zeta_{3}= & \zeta_{5}+6 c_{i, j+1}\left[a_{i, j+1}^{2}\left(\Delta_{i, j+1}-F_{i, j+1}^{t}\right)\right. \\
& \left.+2 a_{i, j+1} c_{i, j+1}\left(F_{i+1, j+1}^{t}-\Delta_{i, j+1}\right)\right], \\
\zeta_{4}= & \zeta_{5}+2 c_{i, j+1}^{2}\left[5 a_{i, j+1}\left(F_{i+1, j+1}^{t}-\Delta_{i, j+1}\right)\right. \\
& \left.+a_{i, j+1} F_{i+1, j+1}^{t}\right], \\
\zeta_{5}= & 2 c_{i, j+1}^{2}\left[\left(a_{i, j+1}+c_{i, j+1}\right)\left(F_{i+1, j+1}^{t}-\Delta_{i, j+1}\right)\right. \\
& +b_{i, j+1}\left(F_{i+1, j+1}^{t}-\Delta_{i, j+1}\right) \\
& \left.-a_{i, j+1}\left(\Delta_{i, j+1}-F_{i, j+1}^{t}\right)\right]
\end{aligned}
$$

and $\sum_{k=0}^{5}(1-\omega)^{5-k} \omega^{k} \zeta_{k}>0$, if $\zeta_{k}>0$ for $k=0, \ldots, 5$. Thus $\zeta_{k}>0$ if

$$
\begin{array}{r}
b_{i, j+1}>\max \left\{0, \frac{c_{i, j+1}\left(F_{i+1, j+1}^{t}-\Delta_{i, j+1}\right)}{\left(\Delta_{i, j+1}-F_{i, j+1}^{t}\right)},\right. \\
\left.\frac{a_{i, j+1}\left(\Delta_{i, j+1}-F_{i, j+1}^{t}\right)}{\left(F_{i+1, j+1}^{t}-\Delta_{i, j+1}\right)}\right\},
\end{array}
$$

or equivalently, for some $s_{i, j}>0$,

$$
\begin{aligned}
b_{i, j+1}= & s_{i, j}+\max \left\{0, \frac{c_{i, j+1}\left(F_{i+1, j+1}^{t}-\Delta_{i, j+1}\right)}{\left(\Delta_{i, j+1}-F_{i, j+1}^{t}\right)},\right. \\
& \left.\frac{a_{i, j+1}\left(\Delta_{i, j+1}-F_{i, j+1}^{t}\right)}{\left(F_{i+1, j+1}^{t}-\Delta_{i, j+1}\right)}\right\}
\end{aligned}
$$

Similarly, the boundary curve $R\left(t_{i}, u\right)$ is convex, if $R^{\prime \prime}\left(t_{i}, u\right)>0$, i.e.,

$$
R^{\prime \prime}\left(t_{i}, u\right)=\frac{\sum_{k=0}^{5}(1-\psi)^{5-k} \psi^{k} \lambda_{k}}{\hat{h}_{j}\left(s_{3}(\psi)\right)^{3}}>0
$$

with

$$
\begin{aligned}
\lambda_{0}= & 2 \hat{a}_{i, j}^{2}\left[\left(\hat{a}_{i, j}+\hat{c}_{i, j}\right)\left(\hat{\Delta}_{i, j}-F_{i, j}^{u}\right)\right. \\
& \left.+\hat{b}_{i, j}\left(\hat{\Delta}_{i, j}-F_{i, j}^{u}\right)-\hat{c}_{i, j}\left(F_{i, j+1}^{u}-\hat{\Delta}_{i, j}\right)\right], \\
\lambda_{1}= & \lambda_{0}+2 \hat{a}_{i, j}^{2}\left[5 \hat{c}_{i, j}\left(\hat{\Delta}_{i, j}-F_{i, j}^{u}\right)+\hat{c}_{i, j} F_{i, j}^{u}\right], \\
\lambda_{2}= & \lambda_{0}+6 \hat{a}_{i, j}\left[\hat{c}_{i, j}^{2}\left(F_{i, j+1}^{u}-\hat{\Delta}_{i, j}\right)\right. \\
& \left.+2 \hat{a}_{i, j} \hat{c}_{i, j}\left(\hat{\Delta}_{i, j}-F_{i, j}^{u}\right)\right] \\
\lambda_{3}= & \lambda_{5}+6 \hat{c}_{i, j}\left[\hat{a}_{i, j}^{2}\left(\hat{\Delta}_{i, j}-F_{i, j}^{u}\right)\right. \\
& \left.+2 \hat{a}_{i, j} \hat{c}_{i, j}\left(F_{i, j+1}^{u}-\hat{\Delta}_{i, j}\right)\right] \\
\lambda_{4}= & \lambda_{5}+2 \hat{c}_{i, j}^{2}\left[5 \hat{a}_{i, j}\left(F_{i, j+1}^{u}-\hat{\Delta}_{i, j}\right)+\hat{a}_{i, j} F_{i, j+1}^{u}\right], \\
\lambda_{5}= & 2 \hat{c}_{i, j}^{2}\left[\left(\hat{a}_{i, j}+\hat{c}_{i, j}\right)\left(F_{i, j+1}^{u}-\hat{\Delta}_{i, j}\right)\right. \\
& \left.+\hat{b}_{i, j}\left(F_{i, j+1}^{u}-\hat{\Delta}_{i, j}\right)-\hat{a}_{i, j}\left(\hat{\Delta}_{i, j}-F_{i, j}^{u}\right)\right],
\end{aligned}
$$

and $\sum_{k=0}^{5}(1-\psi)^{5-k} \psi^{k} \lambda_{k}>0$, if $\lambda_{k}>0$ for $k=0, \ldots, 5$. Thus $\lambda_{k}>0$ if

$$
\begin{array}{r}
\hat{b}_{i, j}>\max \left\{0, \frac{\hat{c}_{i, j}\left(F_{i, j+1}^{u}-\hat{\Delta}_{i, j}\right)}{\left(\hat{\Delta}_{i, j}-F_{i, j}^{u}\right)},\right. \\
\left.\frac{\hat{a}_{i, j}\left(\hat{\Delta}_{i, j}-F_{i, j}^{u}\right)}{\left(F_{i, j+1}^{u}-\hat{\Delta}_{i, j}\right)}\right\},
\end{array}
$$


or equivalently, for some $\hat{r}_{i, j}>0$,

$$
\begin{array}{r}
\hat{b}_{i, j}=\hat{r}_{i, j}+\max \left\{0, \frac{\hat{c}_{i, j}\left(F_{i, j+1}^{u}-\hat{\Delta}_{i, j}\right)}{\left(\hat{\Delta}_{i, j}-F_{i, j}^{u}\right)},\right. \\
\left.\frac{\hat{a}_{i, j}\left(\hat{\Delta}_{i, j}-F_{i, j}^{u}\right)}{\left(F_{i, j+1}^{u}-\hat{\Delta}_{i, j}\right)}\right\} .
\end{array}
$$

Finally, the boundary curve $R\left(t_{i+1}, u\right)$ is convex, if $R^{\prime \prime}\left(t_{i+1}, u\right)>0$, i.e.,

$$
R^{\prime \prime}\left(t_{i+1}, u\right)=\frac{\sum_{k=0}^{5}(1-\psi)^{5-k} \psi^{k} \mu_{k}}{\hat{h}_{j}\left(s_{4}(\psi)\right)^{3}}>0
$$

with

$$
\begin{aligned}
\mu_{0}= & 2 \hat{a}_{i+1, j}^{2}\left[\left(\hat{a}_{i+1, j}+\hat{c}_{i+1, j}\right)\left(\hat{\Delta}_{i+1, j}-F_{i+1, j}^{u}\right)\right. \\
& +\hat{b}_{i+1, j}\left(\hat{\Delta}_{i+1, j}-F_{i+1, j}^{u}\right) \\
& \left.-\hat{c}_{i+1, j}\left(F_{i+1, j+1}^{u}-\hat{\Delta}_{i+1, j}\right)\right], \\
\mu_{1}= & \mu_{0}+2 \hat{a}_{i+1, j}^{2}\left[\left(5 \hat{c}_{i+1, j}\left(\hat{\Delta}_{i+1, j}-F_{i+1, j}^{u}\right)\right.\right. \\
& \left.\left.+\hat{c}_{i+1, j} F_{i+1, j}^{u}\right)\right] \\
\mu_{2}= & \mu_{0}+6 \hat{a}_{i+1, j}\left[\hat{c}_{i+1, j}^{2}\left(F_{i+1, j+1}^{u}-\hat{\Delta}_{i+1, j}\right)\right. \\
& \left.+2 \hat{a}_{i+1, j} \hat{c}_{i+1, j}\left(\hat{\Delta}_{i+1, j}-F_{i+1, j}^{u}\right)\right], \\
\mu_{3}= & \mu_{5}+6 \hat{c}_{i+1, j}\left[\hat{a}_{i+1, j}^{2}\left(\hat{\Delta}_{i+1, j}-F_{i+1, j}^{u}\right)\right. \\
& \left.+2 \hat{a}_{i+1, j} \hat{c}_{i+1, j}\left(F_{i+1, j+1}^{u}-\hat{\Delta}_{i+1, j}\right)\right], \\
\mu_{4}= & \mu_{5}+2 \hat{c}_{i+1, j}^{2}\left[5 \hat{a}_{i+1, j}\left(F_{i+1, j+1}^{u}-\hat{\Delta}_{i+1, j}\right)\right. \\
& \left.+\hat{a}_{i+1, j} F_{i+1, j+1}^{u}\right], \\
\mu_{5}= & 2 \hat{c}_{i+1, j}^{2}\left[\left(\hat{a}_{i+1, j}+\hat{c}_{i+1, j}\right)\left(F_{i+1, j+1}^{u}-\hat{\Delta}_{i+1, j}\right)\right. \\
& +\hat{b}_{i+1, j}\left(F_{i+1, j+1}^{u}-\hat{\Delta}_{i+1, j}\right) \\
& \left.-\hat{a}_{i+1, j}\left(\hat{\Delta}_{i+1, j}-F_{i+1, j}^{u}\right)\right],
\end{aligned}
$$

and $\sum_{k=0}^{5}(1-\psi)^{5-k} \psi^{k} \mu_{k}>0$, if $\mu_{k}>0$ for $k=0, \ldots, 5$. Thus $\mu_{k}>0$ if, for some $\hat{s}_{i, j}>0$,

$$
\begin{array}{r}
\hat{b}_{i+1, j}=\hat{s}_{i, j}+\max \left\{0, \frac{\hat{c}_{i+1, j}\left(F_{i+1, j+1}^{u}-\hat{\Delta}_{i+1, j}\right)}{\left(\hat{\Delta}_{i+1, j}-F_{i+1, j}^{u}\right)},\right. \\
\left.\frac{\hat{a}_{i+1, j}\left(\hat{\Delta}_{i+1, j}-F_{i+1, j}^{u}\right)}{\left(F_{i+1, j+1}^{u}-\hat{\Delta}_{i+1, j}\right)}\right\} \cdot \quad \text { (17) }
\end{array}
$$

Theorem 2 The rational bi-cubic blended function (3) conserves the convexity of surfaces through $3 D$ convex data, if in each rectangular patch $\left[t_{i}, t_{i+1}\right] \times$
Table 2 A 3D convex data generated from function (18).

\begin{tabular}{lccccc}
\hline$t / u$ & -4 & -1.5 & 0 & 1.5 & 4 \\
\hline-4 & -7.98 & -5.47 & -3.96 & -2.39 & 1.01 \\
-1.5 & -5.27 & -2.72 & -1.05 & 1.22 & 14.90 \\
0 & -2.98 & -0.27 & 2 & 6.98 & 59.59 \\
1.5 & 2.06 & 5.48 & 10.46 & 27.56 & 254.67 \\
4 & 55.59 & 69.28 & 113.2 & 304.79 & 3043.6 \\
\hline
\end{tabular}

$\left[u_{j}, u_{j+1}\right], i=0,1,2, \ldots, n-1, j=0,1,2, \ldots, m-1$, the shape parameters are satisfying (11), (13), (15), and (17).

Proof: The result follows immediately from the above discussion.

\section{Algorithm 1}

(i) Given a 3D convex data set $\left\{\left(t_{i}, u_{j}, F_{i, j}\right), i=\right.$ $0,1,2, \ldots, n ; j=0,1,2, \ldots, m\}$.

(ii) Approximate the partial derivatives $F_{i, j}^{t}, F_{i, j}^{u}$, $i=0,1,2, \ldots, n, j=0,1,2, \ldots, m$ at given data points using arithmetic mean method for 3D data proposed in Ref. 3.

(iii) Choose any positive value for free shape parameters $a_{i, j}, a_{i, j+1}, \hat{a}_{i, j}, \hat{a}_{i+1, j}, c_{i, j}, c_{i, j+1}$, $\hat{c}_{i, j}, \hat{c}_{i+1, j}$ and calculate the values of constrained shape parameters $b_{i, j}, b_{i, j+1}, \hat{b}_{i, j}, \hat{b}_{i+1, j}$ using Theorem 2.

(iv) Calculate the rational boundaries $R\left(t, u_{j}\right)$, $R\left(t, u_{j+1}\right), R\left(t_{i}, u\right)$ and $R\left(t_{i+1}, u\right)$ defined in equation (4)-(7) for the rectangular patches.

(v) Put all calculated values from (i), (ii), (iii), and (iv) in the rational bi-cubic blended function (3) to conserve the inherited shape characteristic of 3D convex data.

\section{DEMONSTRATION}

This section deals to examples for demonstration of the developed convex surface scheme for $3 \mathrm{D}$ convex data.

Example 2 The 3D convex data set taken in Table 2 is produced from the following function

$$
F(t, u)=t+u+\mathrm{e}^{t}+\mathrm{e}^{t+u}
$$

Fig. 5 is produced using bi-cubic Hermite spline Ref. 15 that does not conserve the convex surface. To remove this flaw, Fig. 6 depicts the convex surface everywhere in the domain using developed rational bi-cubic blended interpolant with shape parameters $a_{i, j}=\hat{a}_{i, j}=c_{i, j}=\hat{c}_{i, j}=0.25$. 


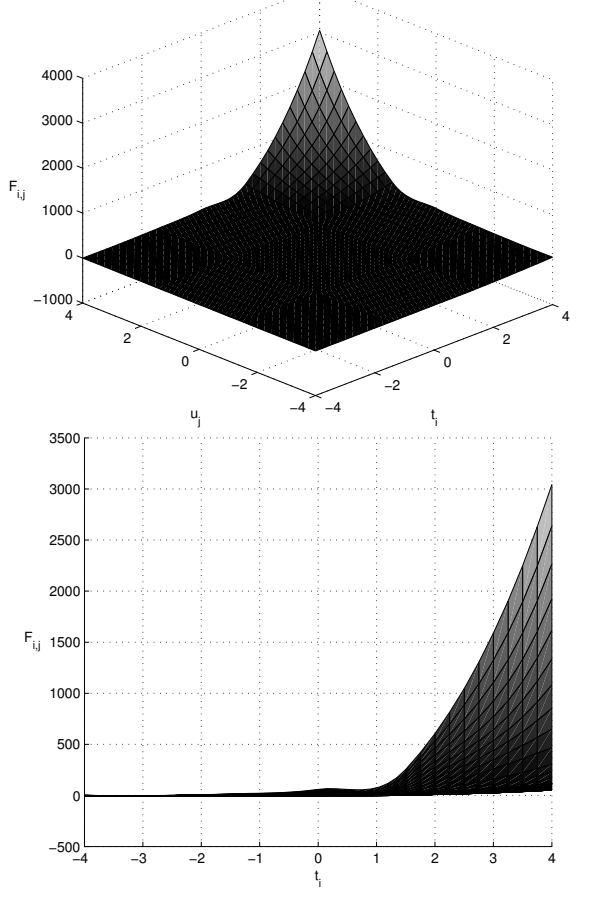

Fig. 5 Bi-cubic Hermite spline surface, and $x z$-view.
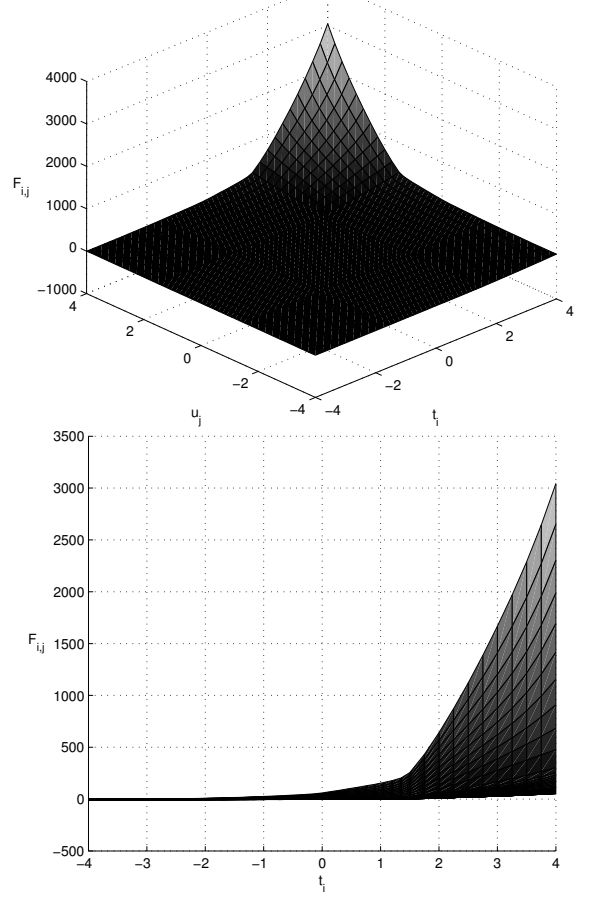

Fig. 6 Convex surface generated by developed rational bicubic blended spline function with $r_{i, j}=\hat{r}_{i, j}=s_{i, j}=$ $\hat{s}_{i, j}=0.01$, and $x z$-view.
Table 3 A 3D convex data generated from function (19).

\begin{tabular}{lccccc}
\hline$t / u$ & 2 & 4 & 6 & 10 & 12 \\
\hline 2 & 101.47 & 100.78 & 100.38 & 99.86 & 99.68 \\
4 & 1443.1 & 1442.4 & 1442.0 & 1441.5 & 1441.3 \\
6 & 7689.6 & 7688.9 & 7688.5 & 7688.0 & 7687.8 \\
10 & $1.16 \mathrm{e} 5$ & $1.16 \mathrm{e} 5$ & $1.16 \mathrm{e} 5$ & $1.16 \mathrm{e} 5$ & $1.16 \mathrm{e} 5$ \\
12 & $5.91 \mathrm{e} 5$ & $5.91 \mathrm{e} 5$ & $5.91 \mathrm{e} 5$ & $5.91 \mathrm{e} 5$ & $5.91 \mathrm{e} 5$ \\
\hline
\end{tabular}
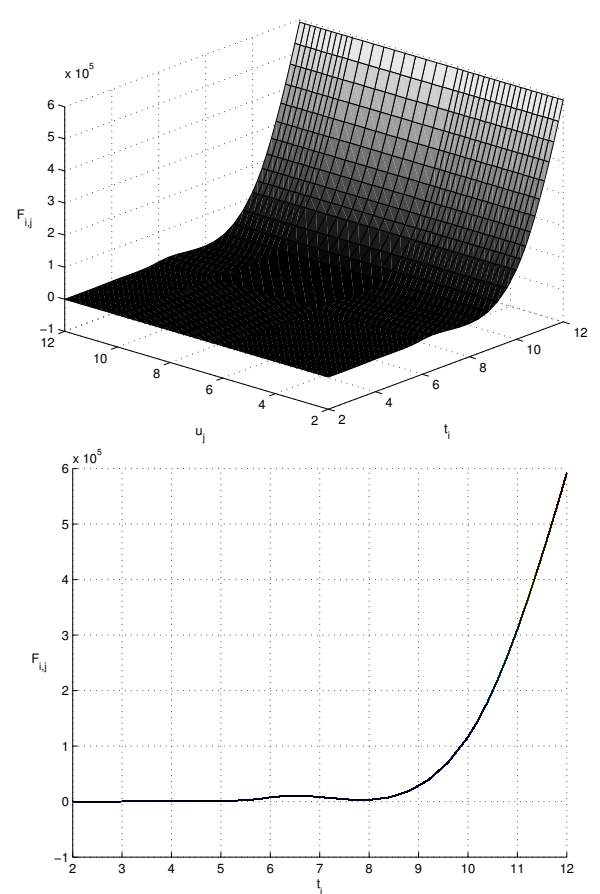

Fig. 7 Non convex surface generated by bi-cubic Hermite spline, and $x z$-view.

Example 3 A 3D convex data set in Table 3 is taken from following mathematical function

$$
F(t, u)=3 \mathrm{e}^{t}+5 t^{4}-\log u
$$

Fig. 7 can be drawn using bi-cubic Hermite spline Ref. 15. It depicts non-convex surface at some knots. On other hand, Fig. 8 can be produced using developed convex rational bi-cubic blended scheme. It conserves the convexity everywhere in the domain with values of shape parameters $a_{i, j}=\hat{a}_{i, j}=c_{i, j}=$ $\hat{c}_{i, j}=0.6$.

Example 4 A 3D convex data set in Table 4 is taken from following mathematical function Ref. 3

$$
F(t, u)=t^{4}+u^{4}
$$

Fig. 9 can be drawn using bi-cubic Hermite spline Ref. 15. It depicts non-convexity preserving surface at 

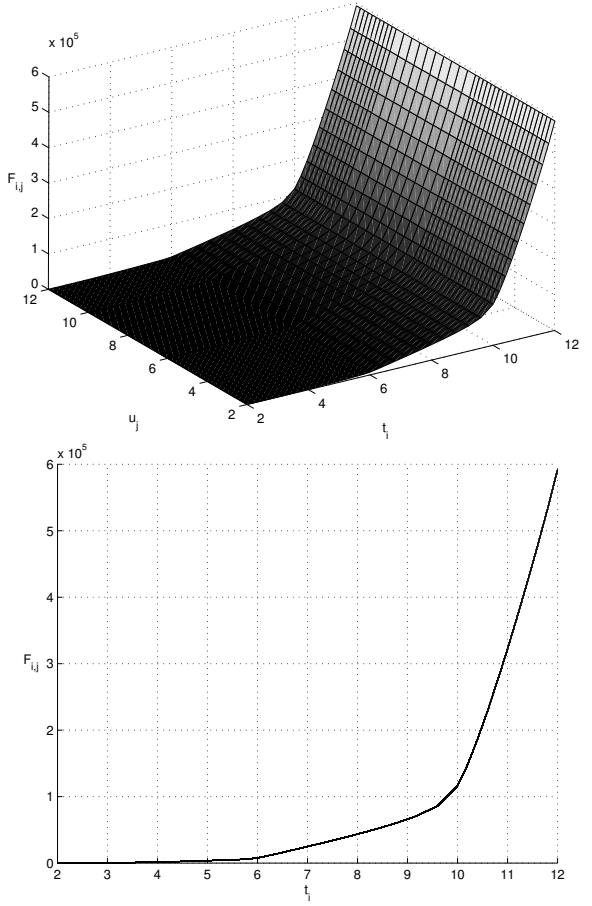

Fig. 8 Convex surface produced by proposed rational bicubic blended spline function with $r_{i, j}=\hat{r}_{i, j}=s_{i, j}=$ $\hat{s}_{i, j}=0.1$, and $x z$-view.

Table 4 A 3D convex data set.

\begin{tabular}{lccccc}
\hline$t / u$ & -4 & -2 & 0 & 2 & 4 \\
\hline-4 & 512 & 272 & 256 & 272 & 512 \\
-2 & 272 & 32 & 16 & 32 & 272 \\
0 & 256 & 16 & 0 & 16 & 256 \\
2 & 272 & 32 & 16 & 32 & 272 \\
4 & 512 & 272 & 256 & 272 & 512 \\
\hline
\end{tabular}

some knots. On other hand, Fig. 10 can be produced using developed convex rational bi-cubic blended scheme. It preserves the convexity everywhere in the domain with values of shape parameters $a_{i, j}=\hat{a}_{i, j}=$ $c_{i, j}=\hat{c}_{i, j}=0.5$.

\section{CONCLUSIONS}

In this paper, we have extended a $C^{1}$ piecewise rational cubic function ${ }^{2}$ to rational bi-cubic blended function to conserve the convexity preserving of surfaces through 3D convex data. The proposed scheme is suitable for such problems in which only data points are given, in contrast the schemes ${ }^{13,14}$ imposed a set of constraints on derivatives and data points, to obtain the convexity preserving surfaces. The developed surface scheme has been demonstrated through several numerical examples and observed that the scheme
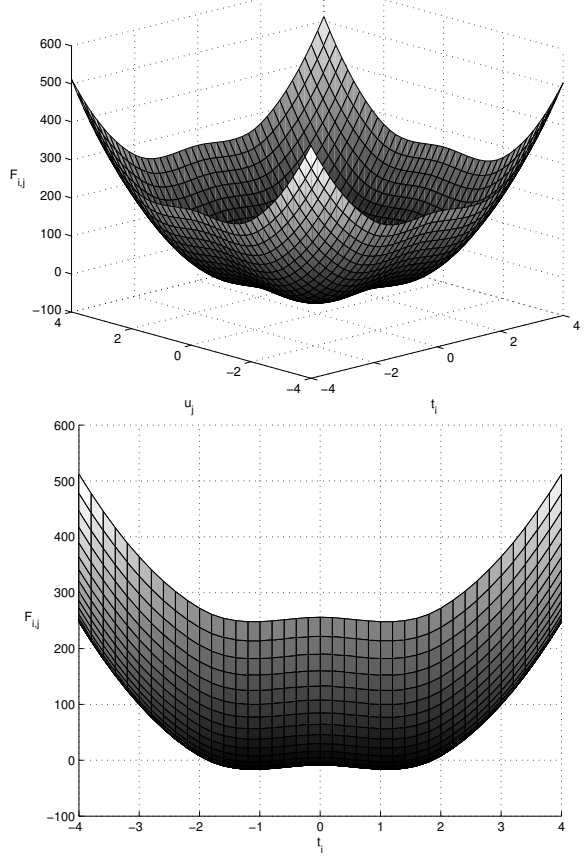

Fig. 9 Non convexity-preserving surface using bi-cubic Hermite spline, and $x z$-view.
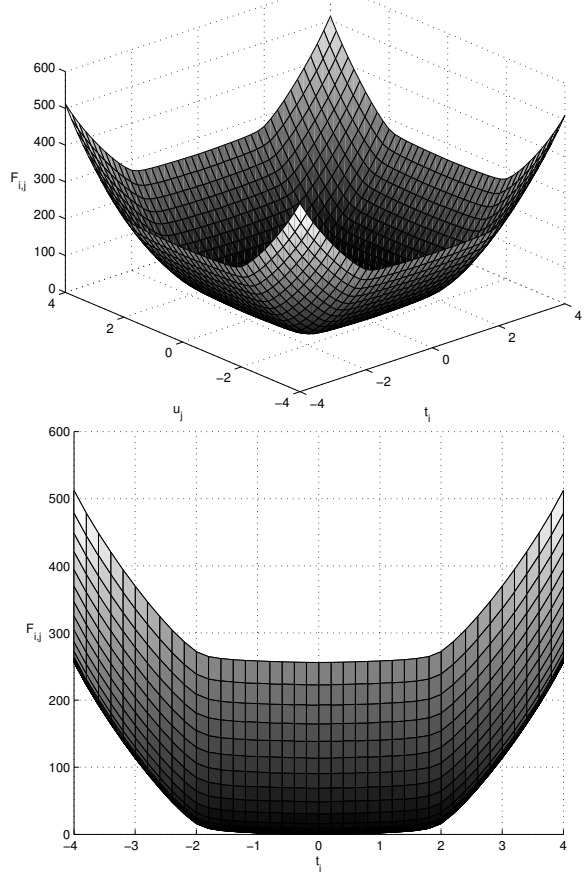

Fig. 10 Convexity-preserving surface using rational bicubic blended spline function with $r_{i, j}=\hat{r}_{i, j}=s_{i, j}=$ $\hat{s}_{i, j}=0.001$, and $x z$-view. 
is not only local and computationally economical but also visually pleasant as compared to existing schemes $^{3,4}$. The proposed surface scheme is unique in its representation and it works well for both uniform and non-uniform space data.

Acknowledgements: We received valuable comments and suggestions from anonymous reviewers to improve this manuscript. The authors are grateful to the financial support from the School of Mathematical Sciences, Universiti Sains Malaysia under the FRGS Grant No. 203/PMATHS/6711324.

\section{REFERENCES}

1. Kuijt F (1998) Convexity preserving interpolationstationary nonlinear subdivision and splines. $\mathrm{PhD}$ thesis, Univ of Twente, Enschede, The Netherlands.

2. Abbas M, Majid AA, Ali JM (2014) Local convexitypreserving $C^{2}$ rational cubic spline for convex data. $S c i$ World J 2014, 391568.

3. Abbas M, Majid AA, Awang MNH, Ali JM (2012) Local convexity shape-preserving surface data visualization by spline function. Br J Math Comput Sci 2 , 72-93.

4. Hussain MZ, Sarfraz M, Shakeel A (2011) Shapepreserving surfaces for the visualization of positive and convex data using rational bi-quadratic splines. Int $J$ Comput Appl 27, 12-20.

5. Roulier JA (1987) A convexity preserving grid refinement algorithm for interpolation of bivariate functions. IEEE Comput Graph Appl 7, 57-62.

6. McAllister DF, Roulier JA (1981) An algorithm for computing a shape-preserving oscillatory quadratic spline. ACM Trans Math Software 7, 331-47.

7. Schumaker LL (1983) On shape-preserving quadratic spline interpolation. SIAM J Numer Anal 20, 854-64.

8. Brodlie KW, Butt S (1991) Preserving convexity using piecewise cubic interpolation. Comput Graph 15, $15-23$.

9. Costantini P (1986) On monotone and convex spline interpolation. Math Comput 46, 203-14.

10. Costantini P, Fontanella F (1990) Shape-preserving bivariate interpolation. SIAM J Numer Anal 27, 488-506.

11. Casciola G, Romani L (2002) Rational interpolants with tension parameters. In: Lyche T, Mazure M, Schumaker LL (eds) Proceedings of Curve and Surface Design, Saint-Malo, Nashboro Press, Brentwood, TN, pp 41-50.

12. Carnicer JM, García-Esnaola M, Peña JM (1996) Convexity of rational curves and total positivity. J Comput Appl Math 71, 365-82.

13. Dodd SL, McAllister DF, Roulier JA (1983) Shapepreserving spline interpolation for specifying bivariate functions on grids. IEEE Comput Graph Appl 3, 70-9.

14. Floater MS (1994) A weak condition for the convexity of tensor-product Bézier and B-spline surfaces. $A d v$ Comput Math 2, 67-80.

15. Farin G (1996) Curves and Surfaces for ComputerAided Geometric Design: A Practical Guide, Academic Press. 